\title{
Lateralization of the Dichotic Digits Test, Central Auditory Processes, and Evoked Potentials in Multiple Sclerosis
}

\author{
Yolanda Peñaloza ${ }^{1,2}$, Martha Valdivia ${ }^{3}$, and Adrián Poblano ${ }^{4}$ \\ ${ }^{1}$ Laboratory of Central Auditory Processing Alterations Research, National Institute of Rehabilitation, Mexico City, México \\ ${ }^{2}$ Postgraduate area, School of Medicine, The National Autonomous University of Mexico, Mexico City, México \\ ${ }^{3}$ Department of Otoneurology, ${ }^{4}$ Laboratory of Cognitive Neurophysiology, National Institute of Rehabilitation, Mexico City, México
}

\author{
Received April 18, 2019 \\ Revised August 7,2019 \\ Accepted August 29, 2019 \\ Address for correspondence \\ Yolanda Peñaloza, MD, MSc \\ Laboratory of Central Auditory \\ Processing Alterations Research, \\ National Institute of Rehabilitation, \\ Calzada México-Xochimilco 289, \\ Col. Arenal Guadalupe, Tlalpan, \\ Mexico City 14389, México \\ Tel/Fax +52-55-5489-6599 \\ E-mail yrpenaloza@inr.gob.mx
}

\begin{abstract}
Lateralization for central auditory processing (CAP) with dichotic digits recognition (DDR) test is believed expression of hemispheric dominance. Multiple sclerosis (MS) is considered an inflammatory and autoimmune alteration of central nervous system (CNS). Hearing alterations in MS and their role in CAP has not been well studied. A patient with MS and new kind of alteration in lateralization of CAP with DDR test is presented. A 53 year of age female with MS of 16 years of evolution, nine of them remained asymptomatic. She has a persistent advantage of the right ear for DDR test; but other monaural tests showed predominance of the left afferent pathway. Brainstem auditory evoked potentials (BAEPs) and long latency auditory evoked potentials (LLAEPS) showed adequate right response with deficits in organization of left response in BAEP, and N2 wave. In the contrary direction of previous publication, we disclosed advantage for DDR test, BAEP, and LLAEP in the right ear. We observed no left ear suppression; with predominance of correct left percentages in monaural psychoacoustics tests. We must keep on searching to find pathophysiological meaning of predominant of right or left auditory laterality as a CAP disorder in patients with MS.

J Audiol Otol 2020;24(1):48-52
\end{abstract}

KEY WORDS: Central auditory processing disorder · Dichotic listening tests Brainstem auditory evoked potentials · Evoked potentials · Multiple sclerosis.

\section{Introduction}

Over the last decades, many authors have demonstrated several central auditory processing disorders (CAPD) in multiple sclerosis (MS) [1,2]. However, it is important for academic and research purposes to continue searching for CAPD features in cases of MS in routine hospital examinations because of its wide clinical variation.

In a previous report, we described differences in auditory lateralization with the dominance of left-sided stimulation in the dichotic digits recognition (DDR) test in patients with MS [3]. However, in the case reported here, we found the opposite behavior; a dominance of right-sided stimulation in

This is an Open Access article distributed under the terms of the Creative Commons Attribution Non-Commercial License (https://creativecommons.org/licenses/by-nc/4.0/) which permits unrestricted non-commercial use, distribution, and reproduction in any medium, provided the original work is properly cited.
DDR, in addition to better organization and shorter latencies in neurophysiological recordings. For this reason, we decided to communicate these results through this case report.

MS has garnered attention from many different viewpoints. We understand much regarding its pathophysiology, diagnosis, therapeutics, and rehabilitation. MS is considered an inflammatory and immuno-mediated alteration of the central nervous system (CNS) [4]. Many reports have given particular importance to cognitive alterations in MS, searching for injuries in the white matter of cortical and nuclear structures, and the formation of abnormal neural connections, according to the different clinical types of MS and their influence on higher cerebral functions, such as auditory perception and working memory, among others $[5,6]$.

The DDR test includes four types of answers: left, right, mixed, and omissions. The total score for the test is obtained from the summation of each type of response and has a max- 
imum of 30 points $[7,8]$.

Lateralization for CAP in the DDR is believed to be the expression of dominant hemispheric auditory function. Thus, the dominance could be left, right, or mixed. The mixed type in the DDR test is selected if left or right responses have the same scores or is predominant itself [8].

Our method for studying CAP included psychoacoustics verbal and non-verbal tests for Spanish-speaking individuals developed at the National Institute of Rehabilitation (INR) and the National Autonomous University of Mexico (UNAM), and have been described elsewhere [3,7,8]. Methods are based on the Bellis model that classifies processing domains alterations as follows: predominantly of the left hemisphere, predominantly of the right hemisphere, of inter-hemispheric relationships, and from the brainstem; the other two types of alterations are those related to attention and receptive language.

In the present case report, we analyzed results from the same psychoacoustics tests in a subject with MS who was not included in the previous report. The patient information was documented in her clinical reports. The second CAPD examination disclosed important alterations in the DDR test, neurophysiological recordings, and neuroimaging studies. Based on the DDR alterations, we propose a close relationship of the DDR test with electrophysiological lateralization anomalies $[7,8]$.

\section{Case Report}

A 56-year-old woman, who works as a store seller, first experienced symptom onset 16 years previously in a first attack that altered tactile sensation in her legs. She was diagnosed with MS and treated accordingly at the Mexican Institute of Social Security and at the INR (Table 1).

The case was studied in the CAPD laboratory in 2011 and 2014. Our study protocol for patients with MS has not changed since the previous publication [4]; however, the method now includes recording of long latency auditory evoked potentials (LLAEPs).

In the interview conducted at 2011, she reported stable tinnitus and bad auditory discrimination since 2010; however, audiograms were normal for both ears. She had a phonemic

Table 1. Clinical outcomes of the patient

\begin{tabular}{|c|c|c|}
\hline Examined function/Procedure & Tests & Results \\
\hline \multirow[t]{4}{*}{ Audiological/Examination } & Pure tone audiometry & Normal range \\
\hline & Speech audiometry & Normal range \\
\hline & Tympanogram & Right: As; Left: A \\
\hline & Acoustic reflex & Right: absent at c500 Hz; Left: normal \\
\hline \multirow[t]{2}{*}{ Otoneurology/ } & Ocular saccades & Dysmetry \\
\hline & Evoked nystagmus & In all directions, but downward or primary position \\
\hline \multirow[t]{5}{*}{ Videonystagmography (2009) } & Pendular tracking & Added nystagmus \\
\hline & Optokinetic test & Right: preponderance \\
\hline & Dix-Hallpike & Right: nystagmus to right traction \\
\hline & Thermal test & $44 \%$ Right-preponderance \\
\hline & & $38 \%$ Left-paresis \\
\hline \multirow[t]{2}{*}{ Laterality/Questionnaire } & Hand & Right \\
\hline & Ear & Right \\
\hline \multirow[t]{6}{*}{ Central auditory processes tests/Battery } & 1st appointment (2011): & \\
\hline & DDR & Right ear advantage \\
\hline & Monaural tests & Normal \\
\hline & 2nd appointment (2014): & \\
\hline & DDR & Right ear advantage \\
\hline & Monaural tests & Left mild advantage \\
\hline \multirow[t]{4}{*}{ Electrophysiology (2014) } & BAEP & Left: bad defined morphology \\
\hline & & Left: retard of waves I, II, and V latencies \\
\hline & & Right: mild I-III retard \\
\hline & LLAEP & Left: retard of N2 wave (246 ms) \\
\hline Neuroimaging & MRI 2009 and 2011 & $\begin{array}{l}\text { Tl, oval images, regular edges, hyperintense, } \\
\text { periventricular location. Some cortical lesions, and } \\
\text { in mesencephalon peduncles and pons }\end{array}$ \\
\hline
\end{tabular}

c500 Hz: contralateral reflex at $500 \mathrm{~Hz}$, DDR: dichotic digit recognition, BAEP: brainstem auditory evoked potential, LLAEP: Iong latency auditory evoked potential 


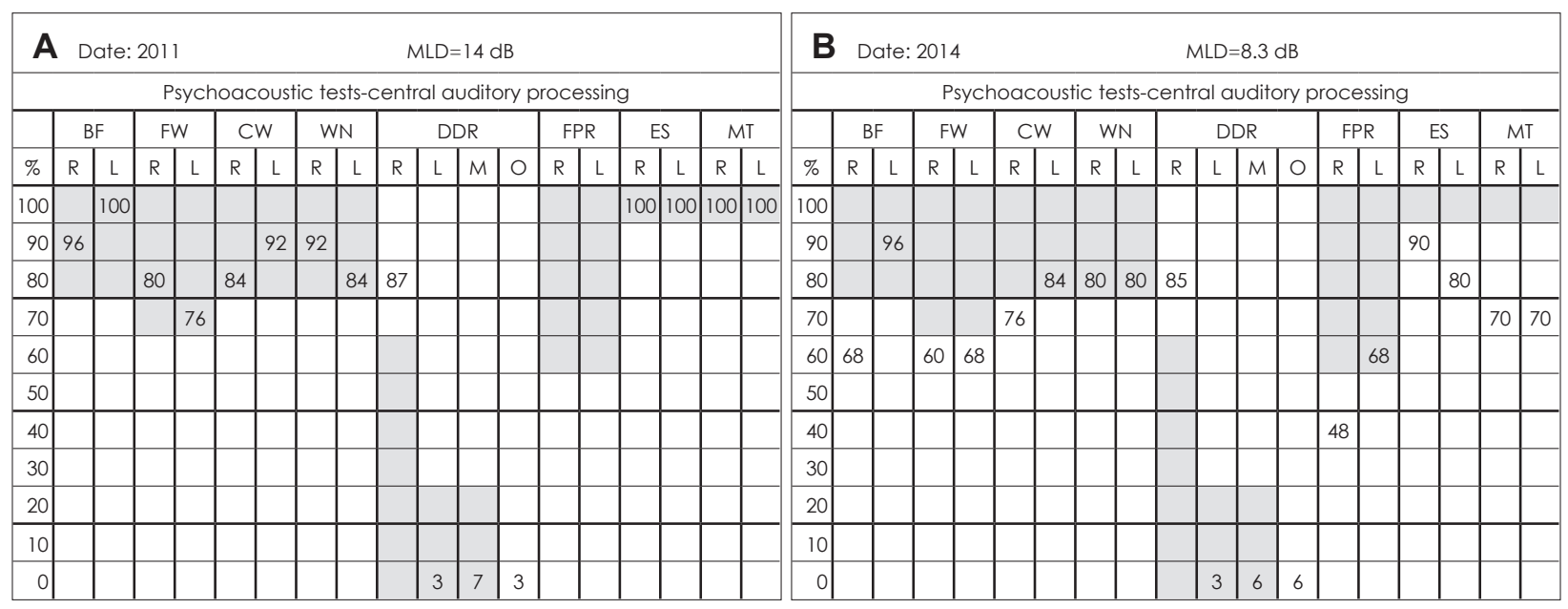

Fig. 1. Results from the studies of psychoacoustics tests-central auditory processes in the two appointments from the case reported. Each box of each column presents the percentage of successes for each test [divided for right $(R)$ and left $(L)$ ear responses]. The left panel (A) shows an adequate result, but right ear advantage in the dichotic digit recognition (DDR) test (2011). The right panel (B) shows poor results, especially in the DDR (2014). Gray area suggests optimal responses. MLD: masking level differences, BF: binaural fusion, FW: filtered words, CW: compressed words, WN: words in noise, FPR: frequency pattern recognition, ES: environmental sounds, MT: music test, M: mixed respoonses, O: omissions.

discrimination deficit, poor sound localization, mixed dysarthria, walking instability with a tendency to fall, and memory disorder (Table 1). The first CAPD study found no significant alterations; she only had a slightly higher percentage of correct answers for DDR test for the right ear (Fig. 1). The study was repeated in 2014 with the same symptoms and persistent tinnitus. Three of four monaural tests showed a dominance of left ear processing, with a higher percentage of successes for the left ear, and a similar profile in the frequency pattern discrimination test. In contrast, the DDR test found a higher percentage of correct answers for the right ear relative to 2011 (Fig. 1).

In brainstem auditory evoked potentials (BAEPs) from the right ear, we observed a mild retardation in interwave interval I-III, and the left ear response presented bad morphology definition and retarded latencies. We also found a retardation of the left N2 wave in LLAEP (Fig. 2). In this case, the results suggest an alteration of the left auditory pathway, probably involving structures connecting temporo-frontal areas, the limbic system, and subcortical thalamic-reticular areas (Fig. 3) [5,7-14], with better function of the right ear in the DDR test, BAEP, and LLAEP.

\section{Discussion}

\section{Main findings and explanations}

The case report here was selected among hospital recordings from the Laboratory of Central Auditory Processes Alterations in 2011 and 2014. The diagnosis of MS was confirmed in two neurology departments of tertiary institutions.
This case is of particular interest because it shows a different result from a previous report, which suggested left ear dominance following stimulation in patients with MS, especially in the DDR test. In the case reported here, the DDR test found a dominance of the right ear.

The DDR test provides information related to the laterality of auditory perception. Nowadays, auditory laterality is considered an expression of basis that is not totally understood $[10,11]$. These facts highlight the particular interest in sharing the results from this case report. The pathophysiological description of MS ranging from imaging, to clinical and evolutionary studies have identified the basic criteria of the disease as being heterogeneous alterations. Thus, it is valid to propose that psychoacoustics tests may show several characteristics, some of them not previously described, and that deserve publication, such as the case reported here.

Relapsing-remitting MS results in a fair model of brain plasticity. Symptoms established in attacks are an expression of neurological alterations; in the next phase, alterations show recovery. However, gradually, the system loses efficiency in recruiting neural networks and the recovery of brain functions. Another relevant fact is the alteration of connectivity as a pathophysiological feature of the disease [6].

The DDR test may show alterations in auditory laterality, even in groups of subjects without evident neurological alterations, such as those with dyslexia [7]. Our laboratory has DDR test result data from many developmental alterations in children and adults such as in peripheral hearing loss, auditory agnosia, and temporal lobe alterations. Similar findings were reported by Martin and Jerger [11] in elderly adults and 
Fig. 2. Electrophysiological tests. (A) Brainstem auditory evoked potentials recordings with clicks at 80 $\mathrm{dB}$, performed in 2014. Left ear with weakly defined wave morphology (upper traces), right ear response with mild retardation in the latency of inter-wave interval I-III (middle and lower traces). (B) Long latency auditory evoked potentials, upper traces with significant retardation of the N2 wave (marked in the traces).

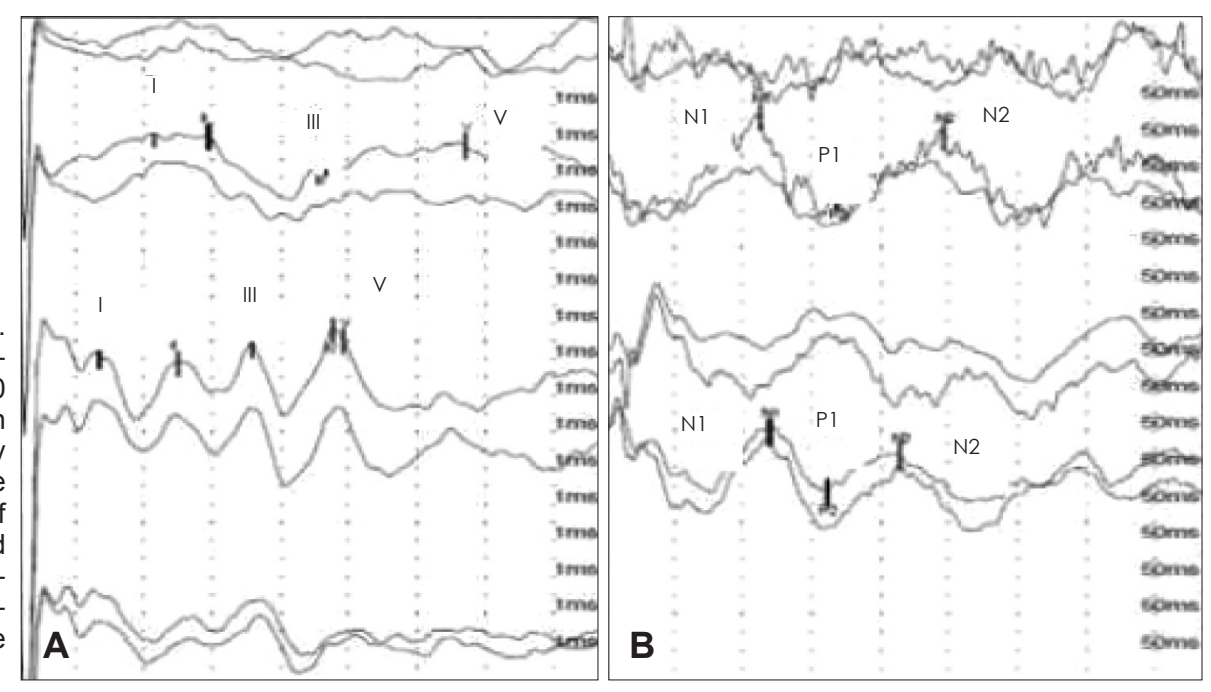

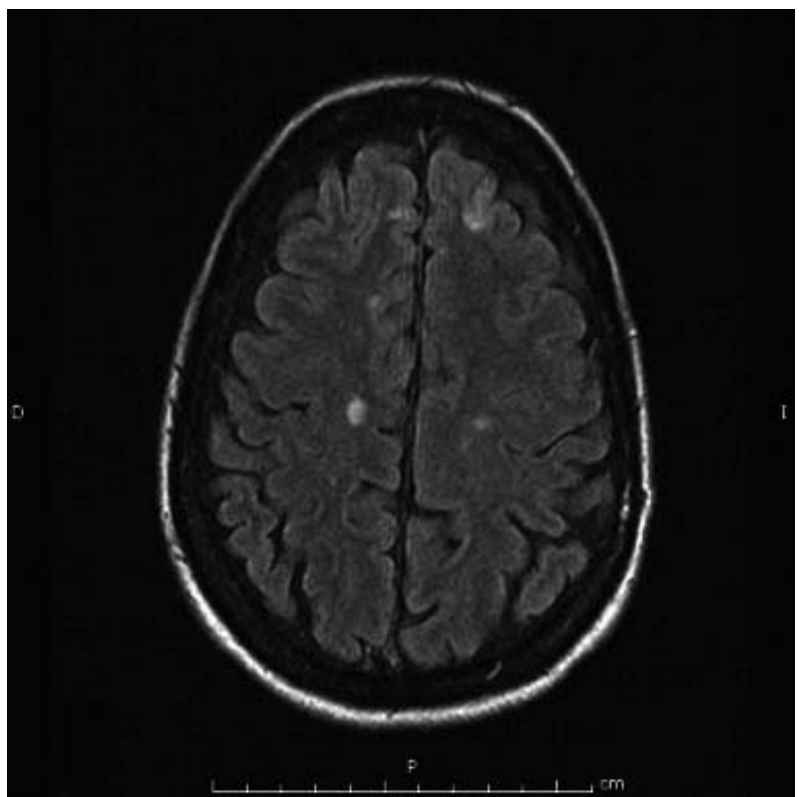

Fig. 3. T1-weighted image with hyperintense oval images with regular edges in the deep white matter, and semi-oval centers in both hemispheres from the patient studied here.

\section{Hendler, et al. [12] in MS.}

The clinical expression in the case reported here may raise questions and controversies regarding the Kimura hypothesis of laterality and predominance of excitatory afferents crossing toward the mesencephalic level and later to the auditory cortex. We expect that the alterations that were initially manifested in BAEP on left-sided stimulation, later made plastic changes in the superior olivary complex that would manifest as a right-sided dominance. In this case, in the BAEP study, some waves were less organized and showed increased wave latencies that were always on the left side; however, we also observed mild retardation of the right I-III interval interwaves.
Reinforcement of right-sided auditory responses was observed in ageing adults and has been related to functional deficiencies in the corpus callosum. This happened when the left auditory pathway, which sends information to the right hemisphere for temporal processing and fine tuning though the corpus callosum to the left hemisphere was injured, and also explained the specific information needed for the articulation pattern following the McGettigan phonetic proposal [9-11]. In this case, although electrophysiological studies are important to identify alterations in the right-sided auditory pathway, there is no functional evidence of effective crossing of auditory information.

\section{Other related studies}

In the medical literature, the relationship between laterality and hemispheric function has been studied, but in fewer reports than the relationship between laterality onset from the brainstem to throughout the cerebral hemispheres. In recent decades, the relationship between cochlear receptor damage and features of hemispheric processing of auditory information has been studied. Tateya, et al. [13] used bad vs. good perception of words, and studied subjects with moderate hearing loss using positron emission tomography. Bad perception words activated the bilateral temporal lobes, inferior frontal gyri, and left angular gyri, with poor ipsilateral activation of the temporal lobe and left angular gyri. The authors established a close relationship between insufficient ipsilateral activation of the temporal lobe with respect to the stimulated side with poor word comprehension performance.

Bergman, et al. [14] studied inter-hemispheric suppression after brain injury, head trauma, and stroke. They used sentences with auditory competence as a dichotic test. The competence sentence exceeded target stimulus intensity and 
evoked frequent alterations in short-term memory when presented to aging adults. Two groups of subjects with hearing thresholds near to normality between $500-2,000 \mathrm{~Hz}$ aged $60-65$ years were studied. They had a $20 \%$ inter-aural difference in psychoacoustic tests, which was considered representative of inter-hemispheric suppression. Observations were made of 34 patients with well-localized brain injury. Right hemisphere injury manifested frequently as complete suppression of contra-lateral perception (left side) using a contra-lateral competence. Left hemisphere brain injury preserved any right-sided advantage [14]. However, it was hard to exclude the possible effects of attention affecting left-sided suppression.

In one case report, a patient with MS was studied pre/posttreatment with cortico-steroid therapy. Authors reported BAEP abnormalities and auditory middle latency response evoked bilaterally, with significant right-sided dominance, and alterations in one of two psychoacoustics tests. The results were suggested to be due to specific brainstem lesions [15].

In our study, we also had an interest in explaining the electrophysiological dominance for the right ear, evident from the first structures of the auditory pathway. We highlight that psychoacoustic mono-aural tests do not show a left-excluding feature; however, the binaural fusion test, which is also a dichotic test, is near to the exclusion criteria, such as the frequency pattern recognition test. These tests, however, show exclusion for the right ear and advantage for the left ear. These data raise the following question: is the right afferent evident in the DDR test, BAEP, and LLAEP a manifestation of constant ipsilateral reinforcement or does this right-sided ipsilaterality influence the possible exclusion observed in the right-sided monaural tests with dominance of processing in the left hemisphere?

Auditory central process examinations in one patient with MS disclosed an advantage for the right ear in the DDR test, which was associated with left ear BAEP and LLAEP alterations. Alterations damage auditory perception and may alter other cognitive functions, such as attention and memory. We must continue searching to find the pathophysiological meaning of CAPD and auditory evoked potentials and their relationships in more patient with MS.

\section{Acknowledgments}

Thanks to Teodoro Flores (MD), Blanca Flores (MD), and Sonia Borja (MD) from the Electrophysiology Department for their help in finding the recordings and their interpretation of neurophysiological studies of the case report. We must also thank the Departments of Audiology, Otoneurology, Phoniatrics, and Neurological Rehabilitation of the INR which provided us their evaluations and orientations regarding clinical, therapeutic, and rehabilitation findings.

\section{Conflicts of interest}

The authors have no financial conflicts of interest.

\section{Author Contributions}

Conceptualization: Yolanda Peñaloza. Data curation: Yolanda Peñaloza and Martha Valdivia. Formal analysis: Yolanda Peñaloza and Martha Valdivia. Investigation: Yolanda Peñaloza and Martha Valdivia. Writing-original draft: All authors. Writing-review \& editing: Adrián Poblano.

\section{ORCID iDs}

Yolanda Peñaloza

Martha Valdivia

https://orcid.org/0000-0002-4758-2867

Adrián Poblano

https://orcid.org/0000-0002-2893-9170

https://orcid.org/0000-0002-1178-8900

\section{REFERENCES}

1) Jerger JF, Oliver TA, Chmiel RA, Rivera VM. Patterns of auditory abnormality in multiple sclerosis. Audiology 1986;25:193-209.

2) Musiek FE, Gollegly M, Kibbe KS, Reeves AG. Electrophysiologic and behavioral auditory findings in multiple sclerosis. Am J Otol 1989; 10:343-50.

3) Peñaloza-López YR, Orozco-Peña XD, Pérez-Ruiz SJ. Multiple sclerosis: left advantage for auditory laterality in dichotic tests of central auditory processing and relationship of psychoacoustic tests with the Mutiple Sclerosis Disability Scale-EDSS. Acta Otorrinolaringol Esp 2018;69:325-30.

4) Cross AH, Naismith RT. Refining the use of MRI to predict multiple sclerosis. Lancet Neurol 2018;17:105-6.

5) Costa SL, Genova HM, DeLuca J, Chiaravalloti ND. Information processing speed in multiple sclerosis: past, present, and future. Mult Scler 2017;23:772-89.

6) Labbe T, Ciampi E, Cruz JP, Zurita M, Uribe S, Carcamo C. Functional magnetic resonance imaging in the study of multiple sclerosis. Rev Neurol 2018;67:91-8.

7) Olivares-García MR, Peñaloza-López YR, García-Pedroza F, Jesús-Pérez S, Uribe-Escamilla R, Jiménez-de la Sancha S. [Identification of auditory laterality by means of a new dichotic digit test in Spanish, and body laterality and spatial orientation in children with dyslexia and in controls]. Rev Neurol 2005;41:198-205.

8) Benavides M, Peñaloza-López YR, de la Sancha Jiménez S, García Pedroza F, Gudiño PK. [Auditory and corporal laterality, logoaudometry and monaural hearing aid gain. Application in symmetric bilateral hearing loss]. Acta Otorrinolaringol Esp 2007;58:458-63.

9) McGettigan C, Scott SK. Cortical asymmetries in speech perception: what's wrong, what's right and what's left? Trends Cogn Sci 2012;16:269-76.

10) Boemio A, Fromm S, Braun A, Poeppel D. Hierarchical and asymmetric temporal sensitivity in human auditory cortices. Nat Neurosci 2005;8:389-95.

11) Martin JS, Jerger JF. Some effects of aging on central auditory processing. J Rehabil Res Dev 2005;42(4 Suppl 2):25-44.

12) Hendler T, Squires NK, Emmerich DS. Psychophysical measures of central auditory dysfunction in multiple sclerosis: neurophysiological and neuroanatomical correlates. Ear Hear 1990;11:403-16.

13) Tateya I, Naito Y, Hirano S, Kojima H, Inoue M, Kaneko K, et al. Inner ear hearing loss modulates ipsilateral temporal lobe activation by monoaural speech stimuli. Neuroreport 2003;14:763-7.

14) Bergman $M$, Hirsch $S$, Solzi P. Interhemispheric suppression: a test of central auditory function. Ear Hear 1987;8:87-91.

15) Schochat E, Matas CG, Sanches SG, Carvallo RM, Matas S. Central auditory evaluation in multiple sclerosis: case report. Arq Neuropsiquiatr 2006;64(3B):872-6. 